\title{
Fluid-phase topology of complex displacements in porous media
}

\author{
Holger Ott $\odot,{ }^{*}$ Ahmad Kharrat, Mostafa Borji $\odot$, and Pit Arnold \\ Department Petroleum Engineering, Montanuniversität Leoben, 8700 Leoben, Austria
}

(Received 7 January 2020; accepted 21 April 2020; published 28 May 2020)

\begin{abstract}
Chemical compositions determine the properties of fluid-fluid and fluid-mineral interfaces and hence the efficiency of multiphase displacement processes in porous media. These interactions are reflected in the microscopic fluid configuration in the pore space, which may be described by topological means. Fluid-phase topology is a promising and currently emerging field of research; however, it still has only a weak link to multiphase displacement physics. We show how the combination of topological and statistical information can be linked to displacement physics and be used for fingerprinting of displacement efficiency and hence to optimize the chemical injection-water composition. We study displacements of crude oil by alkaline injection water in microfluidics, exemplified by other water-based chemical methods. A complex coupling of fluid flow and fluid-phase behavior has been observed with the formation of emulsion phases during displacements. Oleic phases were analyzed by statistical and topological means, showing a systematic change as a function of alkali concentration linked to emulsification. In particular, Lorenz diagrams and a scaled Euler characteristic have been linked to physical properties and fluid-phase behavior and have been found to be sensitive to changes in injection-water chemistry.
\end{abstract}

DOI: 10.1103/PhysRevResearch.2.023240

\section{INTRODUCTION}

Displacements of nonaqueous phase liquids (NAPLs) by chemically modified water-based fluids may be complex due to the coupling of multiphase flow, chemical interactions, and the resulting phase behavior of the fluids. The quantification and optimization of the displacement efficiency in such cases is a long-standing issue. Technically and environmentally important NAPLs may be crude oils, refined oils, or hydrocarbon-based fuels in hydrocarbon reservoirs; contaminated fresh-water aquifers; or contaminated soils. The challenge in all these cases is to clean, respectively, displace, NAPLs trapped in the pore space of rocks or soils. Surfactantbase infiltration has been identified as a suitable method [1-4].

Reservoir engineers developed several chemical techniques to displace oil from porous rocks. Alkaline flooding, the present example, is one of them and is considered a chemical enhanced oil recovery (EOR) method, which is often combined with surfactant and polymer flooding $[2,5,6]$; the addition of an alkaline agent to the injection water results in a high $p \mathrm{H}$ and can lower the interfacial tension between an oleic and aqueous phase when the crude contains a substantial amount of fatty acids, which is reflected in the total acid number (TAN). At high $p \mathrm{H}$ values, hydroxide ions and acidic oil components saponify at the oil-water interface, resulting in the generation of in situ surfactants $[2,7,8]$. The resulting decrease

\footnotetext{
*holger.ott@unileoben.ac.at

Published by the American Physical Society under the terms of the Creative Commons Attribution 4.0 International license. Further distribution of this work must maintain attribution to the author(s) and the published article's title, journal citation, and DOI.
}

in interfacial tension commonly leads to emulsification and an increasing displacement efficiency, leading to additional oil recovery. Alkaline flooding is in those cases a highly effective, low-cost, and environmentally friendly solution.

Complex chemical EOR processes such as alkali or surfactant flooding are typically optimized for their phase behavior and by conventional core flooding experiments [9]. However, the information from core floods is rather limited, as there are no direct insights into details of oil mobilization and displacements. As a rule, cumulative oil production and differential pressure are measured. This is rather one-dimensional information from which complex displacement processes must be understood. On the other hand, the phase behavior, respectively, the degree of emulsification, is often analyzed in test tubes and not under realistic flow, respectively, mixing conditions as in porous media.

Initial NAPL mobilization takes place in the rock's/soil's pore space and there are several techniques by which this can directly be observed, such as micro $\mathrm{x}$-ray computedtomography (micro-CT) imaging [10-12] and microfluidics [13-16]. It has recently been demonstrated that both techniques, and especially microfluidics, allow as well the identification of emulsion phases and hence give access to combined information on flow and phase behavior [14,16].

In recent years and with the development of pore-scale imaging methods, fluid distributions have been analyzed by statistical and topological means, deriving information that is more sophisticated and may provide more insight into displacements physics than, e.g., recovery curves and differential pressure measurements alone; if a fluid phase forms ganglia or isolated clusters, cluster-volume and cluster-length distributions have been used to characterize fluid phases and attempts to link these distributions to fluid-phase mobility has been made $[10,11,17-19]$. These distributions are characteristic, 
but their interpretation with respect to fluid-phase mobility still lacks fundamental understanding and may still be scale dependent [18].

Another very recent development is the description of the microscopic fluid-phase distribution by topological means using Minkowski functionals [20,21]. Fluid-phase topology is linked to classical parameters such as fluid saturation, capillary pressure, capillary pressure hysteresis, and the wetting state $[21,22]$. With the correlation of the Euler characteristic to relative permeability, a first link to fluid dynamics has been found [23]. The advantage of a topological description in general is that it assigns these parameters a well-defined physical and mathematical meaning. Of particular interest in this context is the Euler characteristic, which describes the connectivity of a fluid phase and may be associated with wetting properties [21]. In a further step, integral geometry may be used for a thermodynamic description of fluid in porous media in order to develop next-generation two-phase flow models $[24,25]$.

In the present study, we show that the efficiency of displacements can be evaluated by topological properties of the microscopic fluid phase. For this, we link integral geometry to system properties like wettability, connectivity, recovery, and recovery potential of NAPLs applying statistical methods.

The example of use is the displacement of crude oil by alkali solutions of different concentrations. We use microfluidics (MF) to screen the performance of the injection water as a function of alkali concentration; the advantage of using MF devices is that they are chemically well defined, experiments can efficiently be performed, and fluid displacements can be imaged with relatively high spatial and time resolution. There are of course shortcomings with respect to interactions of fluids with mineral phases and with mimicking realistic rock structures. In the present work, we focus on fluid-fluid interactions and use therefore MF according to its strength; we take advantage of the high spatial and temporal resolution achievable in MF to observe fluid displacement processes such as ganglion dynamics, breaking of oil clusters, changes of wetting states, and the formation of emulsion phases. We analyze fluid-phase distributions by means of a statistical and topological description of oil clusters, and link the observations to the injection-water chemical composition.

The study shows that there are topological signatures of complex displacement processes. Those can be applied to evaluate displacements and to screen the efficiency of chemical water compositions. This may be a step toward statistical/topological fingerprinting of displacements. Especially in cases of emulsion formation, the evaluation of fluid topology is not anymore unique and may be a matter of interpretation depending on the exact physical question that needs to be addressed. Those interpretations provide a link between the mathematical description and physical system properties.

\section{MATERIALS AND METHODS}

Porous medium. The microfluidic chips were made from borosilicate glass, representing a two-dimensional (2D) porous medium. The pattern has a homogeneous etching depth of $20 \mu \mathrm{m}$ and a lateral definition of pores and pore throats. Porosity and permeability are $\phi=0.57$ and $K=$
$2.5 \mathrm{D}\left(1 \mathrm{D} \approx 10^{-12} \mathrm{~m}^{2}\right)$ and total pore volume to $2.3 \mu 1$. The total porous domain size is of $20 \times 10 \mathrm{~mm}^{2}$ with channel systems at the inlet and outlet for fluid distribution and fluid collection. The porosity-based representative elementary volume (REV) is $5 \times 5 \mathrm{~mm}^{2}$. The lateral porous structure is rather open and homogeneous with all features larger than the etching depth.

Instrumentation. The experiments were performed at ambient conditions with a constant flow rate boundary condition at the inlet, and a constant pressure boundary condition at the outlet. Fluids were injected with a high-precision syringe pump. For each fluid type, a separate syringe was used. On the downstream side, fluids were produced to atmospheric pressure. For preparation purpose, a vacuum pump was installed on the downstream side.

For imaging the pore space, a Leica DMi8 microscope with a wide-range automated $x y$ table and an image stitching option was used, providing a high spatial resolution and a time resolution that allowed for detailed investigation of displacement processes and fluid-phase configuration. The images were recorded with a Leica DMC2900 camera.

Fluids. The oil sample is derived from an oil field in the Vienna basin. The sample was characterized by viscosity, $\mu=330 \mathrm{cP}$ at $20^{\circ} \mathrm{C}$, interfacial tension to water (IFT) $\sigma=$ $0.7 \pm 0.1 \mathrm{mN} / \mathrm{m}$ at $20^{\circ} \mathrm{C}$, and the total acid number (TAN) $1.96 \mathrm{mg} \mathrm{KOH} / \mathrm{g}$ oil. As injection water, de-ionized (DI) water was used. Alkaline solutions were prepared on the basis of DI water with $\mathrm{Na}_{2} \mathrm{CO}_{3}$ as alkali agent in concentrations in between 200 and $12000 \mathrm{ppm}$. In this range, the $p \mathrm{H}$ varies monotonically in between 10.6 and 11.6.

Sample preparation. Before each experiment, the microfluidic chips were cleaned by injecting 250 pore volumes (PVs) of distilled water at high flow rate and subsequently a sequence of solvents at low flow rate to allow for efficient displacement and mixing with the fluids in place: $50 \mathrm{PV}$ of acetone followed by $50 \mathrm{PV}$ of toluene followed by another $50 \mathrm{PV}$ of acetone to clean out the toluene. In a final step, vacuum was applied to vaporize the acetone. After cleaning, oil was injected under vacuum conditions until the sample was oil saturated-no connate water was present at the beginning of the experiments.

Flooding experiments. The starting point of each experiment was a fully oil saturated sample $\left(S_{o}=1\right)$. To mimic a tertiary recovery process, first water was injected immiscibly displacing oil from the porous domain (secondary recovery). The flooding time was $24 \mathrm{~h}$, longer than the time to reach steady state with no further detectable change of the oil saturation in the porous medium. Subsequently, the samples were flooded with alkaline solutions in order to study tertiary displacement effects. The alkaline-flooding time did not exceed $24 \mathrm{~h}$ either. For both water and alkaline flooding, an injection rate of $0.01 \mathrm{ml} / \mathrm{h}$ was chosen, leading to an interstitial velocity of about $2.4 \times 10^{-5} \mathrm{~m} / \mathrm{s}(\sim 6.9 \mathrm{ft} /$ day $)$. The total injected volume corresponds to approximately 100 PV. This relatively high flow rate corresponds to velocities in the vicinity of injection wells, where initial oil mobilization may take place prior to oil-bank formation.

Optical images of the flood domain were taken under flow conditions; in some cases, with a relatively small field of view 
and a high time resolution of about $100 \mathrm{~ms}^{-1} \mathrm{~s}$, in most cases with a field of view that covered the entire flood area with high spatial resolution, resulting in a lower time resolution of the order of $30 \mathrm{~s}^{-1} \mathrm{~min}$.

Uncertainties. Calculations of areas consider a segmentation error referring to the gray-scale definition of the fluidfluid and the fluid-grain boundaries. Artifacts at boundaries were removed by applying an erosion-dilation process. Therefore, we consider the error of areas as in the order of the perimeter as given in units of pixels in Fig. 5, which is in the order of the extension of the respective symbols in the graphs. A further source of error is the optically unresolved W/O emulsion phases; emulsification leads to a swelling of the oleic phase and hence to an overestimation of oil recovery. From observations, we estimate the error by oil swelling of individual bubbles to be $<10 \%$ (maximum observed swelling of an insulated droplet was $20 \%$ after $40 \mathrm{~h}$ flooding), and for saturation calculations to be $<1 \%$ (swelling multiplied by affected saturation), which we therefore neglect.

The Euler characteristic and the cluster volume distributions are affected in cases where emulsion phases form. The difficulty is how to deal with optically resolved features that may be considered as texture of an emulsion phase, or alternatively as individual features to be taken into account for topological description. Texture turns out to be a matter of interpretation and is discussed in the text.

Further uncertainty is associated with the repeatability of individual experiments as included in Fig. 5. Data on water flooding $(0 \mathrm{ppm})$ scatter due to the dominance of large clusters that are not well represented in the porous domain [18]. Saturation-based REVs were measured; higher-concentration alkali experiments have shown that the REVs are below systems size (SS). The REVs of water floods were on the order of the SS (REVs $\geqslant$ SS cannot conclusively be proven). However, oil phase texture and Lorenz diagrams show robust repeatability [26].

\section{OBSERVED ELEMENTARY PROCESSES}

Experiments were performed in microfluidics, i.e., 2D artificial porous media etched in borosilicate glass representing a highly permeable relatively open pore structure. Figure 1 shows microscopic images of a subsection of the porous domain during an experimental sequence. The initial state of all experiments is a fully oil saturated sample with the oil saturation $S_{o}=1$ [left image of series (a); the "grains" of the pore structure appear in light gray and the oil saturated pore volume in dark gray. To study tertiary displacement processes, the samples are at first water flooded [Fig. 1(a)] to a state close to residual saturation $\left(S_{o} \approx S_{o r}\right)$ at which the oleic phase is practically immobile. The remaining oil is the target of the subsequent alkaline flooding [Fig. 1(b)].

Water flooding. During the preceding water flood, water invades by "percolating" through the pore space, immiscibly displacing oil. Some larger fraction of the oil may be bypassed by the percolating water and may be disconnected forming insulated clusters or ganglia. At a later stage, the dynamics of these ganglia appears to dominate the flow of oil, which is illustrated in Fig. 1(a). Where the injection water comes in contact with the solid phase, the wetting condition changes from the initial oil-wet to a water-wet state as observed by the contact angle at the three-phase contact points, a process that is known from low-salinity water flooding [27,28]; the resulting overall wetting state may be described as mixed wet, with the remaining larger clusters or ganglia being pinned to the grains.

Over time, larger clusters break up in smaller, nonwetting droplets/clusters by viscous forces (snap-off). Those clusters are mobile, flow with the water stream, and are ultimately recovered. After a certain time, a steady state is reached, where the viscous forces that the water flow applies to the remaining oil phase are in equilibrium with the trapping forces, i.e., capillary and adhesion forces. At this point, the remaining oil phase is stationary and $S_{o} \approx S_{o r}$. In this specific experiment, $52 \%$ of the oil has been recovered. The changes in flow regimes form connected pathways to ganglion dynamics to drop-traffic flow can be linked to the relativephase-permeability saturation function as earlier observed by Avraam and Payatakes [29].

Alkaline flooding. During the subsequent injection of an alkalic solution [Fig. 1(b)] capillary forces are lowered by lowering the interfacial tension between oil and water. During this process, pinned oil clusters break down further in smaller fragments by the applied viscous force. The smaller fragments are predominantly nonwetting and mobile, leading to a droptraffic flow until a new steady state at a lower oil saturation is reached. In the example given in Fig. 1(b), 72\% of the previously remaining oil was recovered. The experiment is representative for optimum conditions. Outside optimum alkali concentration, the flow is influenced by complex phase behavior as will be discussed in the following.

Effects of emulsification. As the high-TAN oil comes in contact with an alkalic solution, the interfacial tension is lowered to a degree such that emulsions may form. This is shown in Fig. 2 for a series of experiments with different $\mathrm{Na}_{2} \mathrm{CO}_{3}$ concentrations in test tubes; the same volumes of crude oil and alkali solution were brought in contact with each other, the fluids were mixed by shaking the tubes, and subsequently put at rest for 4 weeks. At rest and with time, the fluids separate by gravity to their equilibrium state and stable fluid phases were observed. ${ }^{1}$ In the present case, oil-in-water $(\mathrm{O} / \mathrm{W})$ and water-in-oil (W/O) emulsions have been observed simultaneously by the coloring of the aqueous and oleic phases, and a nonmonotonic level change of the oil-water contact is visible. The behavior is quantified by computedtomography (CT) scans, which show the interface position as well as the degree of mutual phase participation. We observe oil phase swelling with $C_{\mathrm{Na}_{2} \mathrm{CO}_{3}}$ before and after optimum conditions at 3000 ppm as displayed in the panels (c)-(e). For optimum conditions, the system returns to close to its original density and level values and a third phase occurs, which may be interpreted as the microemulsion phase. The progression is discussed in the frame of Fig. 5.

In the described test-tube experiments, the mixing regime was turbulent and the phase behavior was observed in

\footnotetext{
${ }^{1}$ The dynamics is very slow; the microemulsion phase has been observed after 4 weeks. Visual observations after $48 \mathrm{~h}$ are as reported in [26].
} 


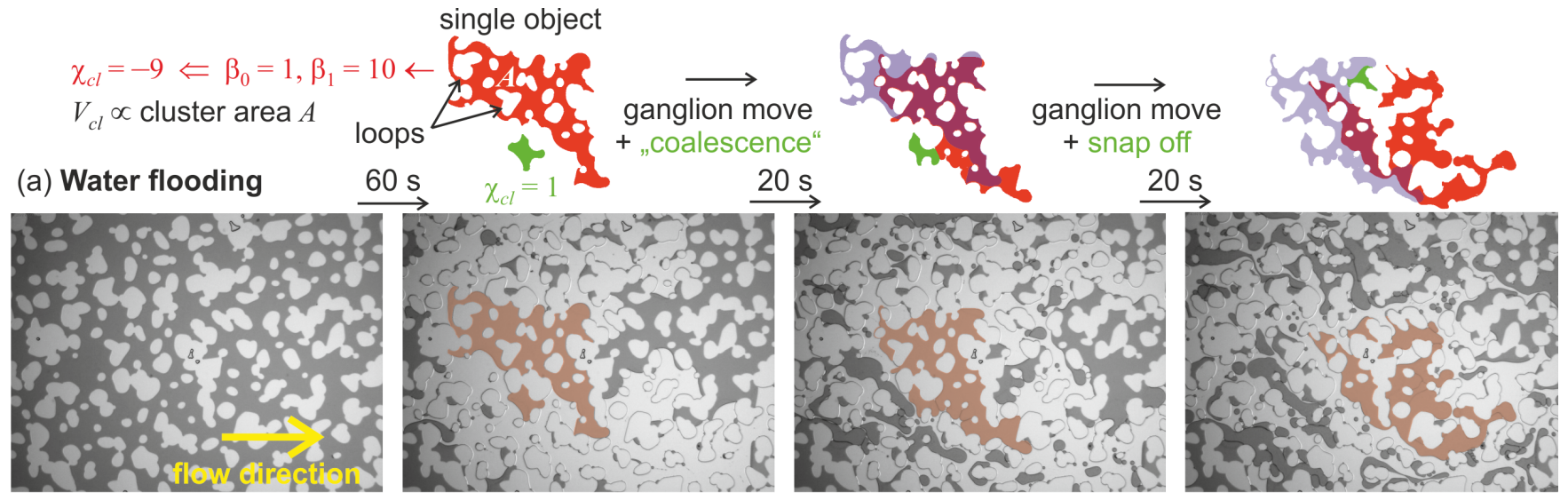

(b) Alkaline flooding $\sim 6 \times 10^{3} \mathrm{~s}$

$\stackrel{20 \mathrm{~s}}{\longrightarrow}$

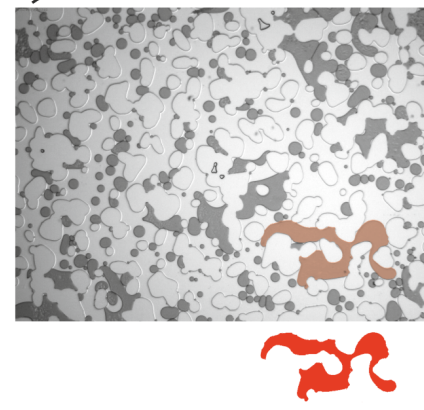

(c) After water flooding; segmented total domain

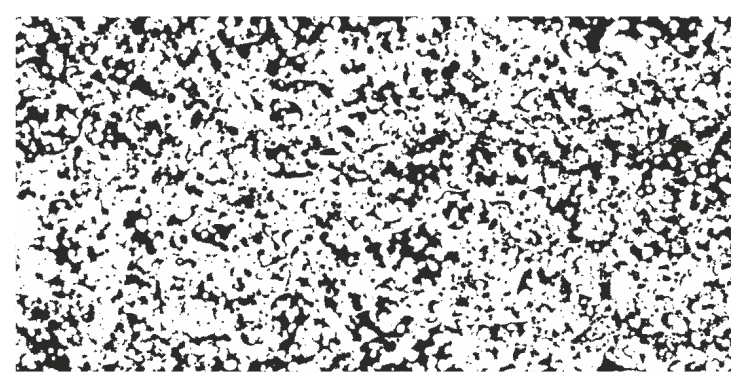

$\stackrel{\sim 3 \times 10^{5} \mathrm{~s}}{\longrightarrow}$
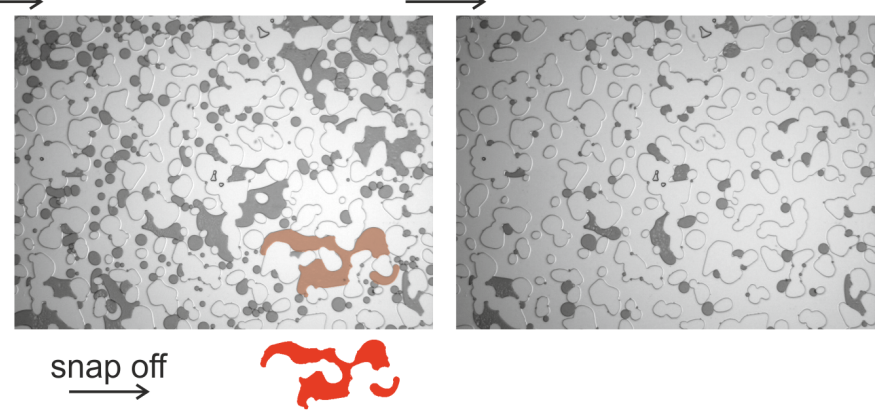

(d) After alkaline flooding; segmented total domain

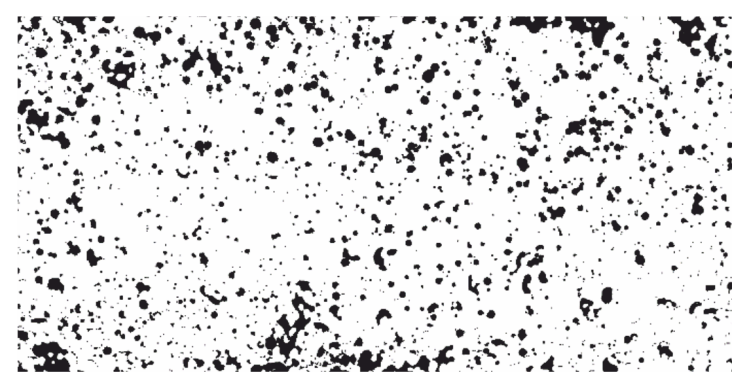

FIG. 1. Time series of elementary displacement processes during water flooding (a) and subsequent alkaline flooding with a 950-ppm $\mathrm{Na}_{2} \mathrm{CO}_{3}$ aqueous solution (b). The flow direction is from left to right. Saturation-state changes and drop-traffic flow are best recognized by viewing adjacent images as stereo images (try it out!). Extracted clusters outside the images illustrate elementary processes and aspects of the topological analyses. Segmented oleic phase after water flooding (c) and alkaline flooding (d). Such images are the basis for the topological and statistical analyses presented below.

equilibrium. This is not necessarily representative for porous medial flow, which is typically considered to be laminar at realistic flow conditions as applied in this study. There are a couple of important and noteworthy observations: In contrast to the test tube experiments, in porous media flow, only a very small volume of emulsion was formed. Emulsification was predominantly observed for rather low and high alkali concentrations but not, or just to, a minor extent in between, close to the observed optimum.

W/O emulsions may be classified by their droplet size as loose - the optically resolved — and tight emulsions [30]. Tight emulsions appear as the homogeneous phase and darker as the initial oil phase, becoming darker and expanding in time. As illustrated in Fig. 3, the gray scale between emulsions and the oil phase changes gradually and distinguishing between oleic phases by phase segmentation may not be useful. With the below applied two-phase segmentation approach, we partly ignore emulsion phases. This is because water participates in emulsions to a certain volume, which we account for as the oleic phase-the oil phase is swelling. As a consequence, oil saturations may be overestimated, which leads to an error in the recovery calculations that we estimate to be below $1 \%$. On the other hand, emulsions have been found to pin oil clusters, i.e., to demobilize the oleic phase; because emulsification influences cluster mobility, it is implicitly accounted for in the cluster topology and cluster statistics as given further below.

Conglomerates. During displacements, mobile oil clusters may come into contact with each other. Depending on interfacial properties they may merge forming larger clusters. This has been observed in several pore-scale displacement studies using model oils $[10,11,31]$. Such coalescence events have 

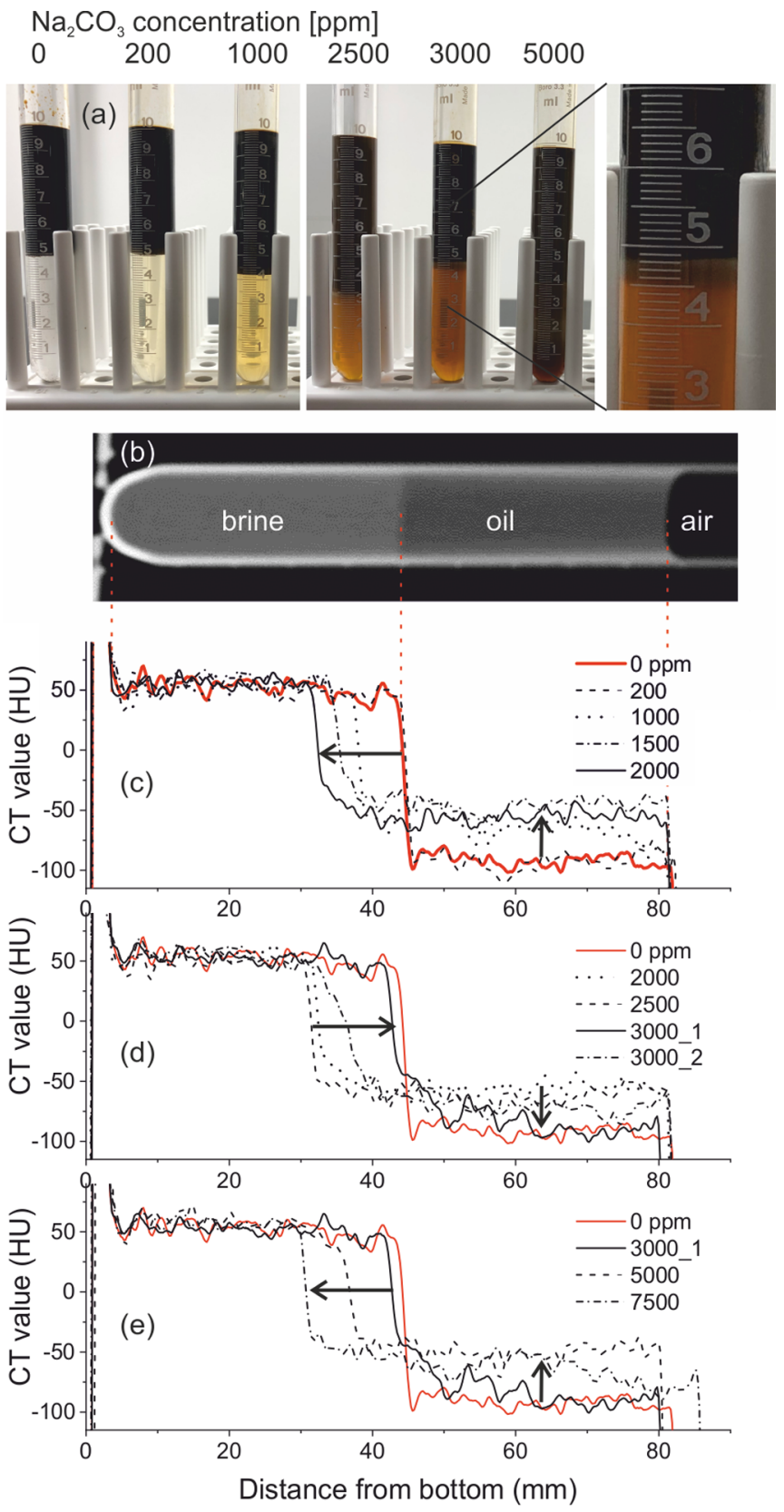

FIG. 2. Phase behavior after mixing crude oil with water of different $\mathrm{Na}_{2} \mathrm{CO}_{3}$ concentrations in ppm. (a) Photographs of the test tubes showing the nonmonotonic behavior, and the appearance of a third phase at a concentration of 3000 ppm. (b)-(e) Evaluation of the phase behavior by the density profiles obtained from computer tomography (CT). The data show the complex nonmonotonic oil swelling behavior with alkali concentration. The CT contrast between the oleic and aqueous phase and the shift of the oil-water contact is summarized in Fig. 5.

been observed for higher alkali concentrations (i.e., lower interfacial tension) in the current study. Sometimes, especially at low alkali concentrations, bubbles adhere together without merging. This is illustrated in Fig. 3(a) for the 200-ppm case. Such conglomerates are stable configurations of oil droplets separated by dark-appearing interfaces. Such conglomerates might be interpreted as oil-in-water $(\mathrm{O} / \mathrm{W})$ emulsion. Even appearing to the eye as individual droplets, conglomerates may behave as large individual clusters. Depending on rtheir physical behavior, topologically, such structures may be interpreted as single entities or as individual objects, which is discussed in the topological description further below.

\section{TOPOLOGICAL AND STATISTICAL SIGNATURES}

Recovery factor. The purpose of water injection is to recover NAPL or crude oil from porous media such as soils or reservoir rocks. The respective recovery factors of the secondary water floods, $R_{\mathrm{sec}}$, were calculated from the remaining oil in the pore space, represented by the dark area, $A_{\text {oil }}$, after image segmentation: $R_{\mathrm{sec}}=1-\frac{A_{\mathrm{oil}}}{A_{\text {pore }}}$, where $A_{\text {pore }}$ corresponds to the porosity, which is measured from the initial state, $S_{o}=1$, in the same way as later $A_{\text {oil }}$. For tertiary floods, we calculate the additionally recovered oil $R_{\text {tert }}$, which is defined as $R_{\text {tert }}=1-\frac{A_{\text {oil }}}{A_{\text {oil.sec }}}$, and which refers to the volume of oil in place after the preceding water flooding.

The recovery curves show differences in the displacement efficiency for different $\mathrm{Na}_{2} \mathrm{CO}_{3}$ concentrations; in Fig. 4(a), $R_{\text {tert }}$ is plotted as a function of time. Different ultimate recoveries as well as different time dependencies were observed, while, e.g., at 1000 and 3000 ppm similar ultimate recovery factors were reached; the 3000-ppm recovery curve appears steeper and ultimate recovery was reached much earlier. Above and below this range, ultimate recovery decreases.

On average, the recovery factor achieved by water flooding varies over the experiments with an average of $0.43( \pm 0.1)$ with a standard deviation of 0.1 . The variation is a result of the relatively small flooding domain, which is larger than the porosity-based representative elementary volume (REV), but not necessarily as the saturation-base REV; it is known that the REV two-phase flow depends on the distribution of the fluids in the porous domain [18].

Figure 5(b) shows the final tertiary recovery factor as a function of $\mathrm{Na}_{2} \mathrm{CO}_{3}$ concentration. According to the definition, at zero concentration, no additional oil is recovered. With increasing $\mathrm{Na}_{2} \mathrm{CO}_{3}$ concentration, the recovery factor increases steeply with a maximum in the range between 1000 and $3000 \mathrm{ppm}$. At higher concentrations, the recovery factor decreases again. This behavior will be further characterized in terms of wettability and is affected by emulsion formation. After alkaline flooding, the oil cluster sizes are generally smaller, and the REV is therefore generally smaller than for the respective water floods and in the present case smaller than the porous domain.

Minkowski functionals. The segmented images provide more information than just the oil saturation from which we calculated the recovery factor, i.e., the efficiency of the displacement process. Minkowski functionals can be used for an integral view on the oleic-phase topology. Since microfluidics provides 2D information, there are three Minkowski functionals $(D+1)$ for a full description of the fluid-phase topology, which are the area $A$, the perimeter $U$, and the Euler characteristic $\chi$ [32]. Figure 5(a) shows the MF of the respective steady-state fluid configuration as a function of alkali concentration. The results on the prior water flooding 
(a) $200 \mathrm{ppm}$

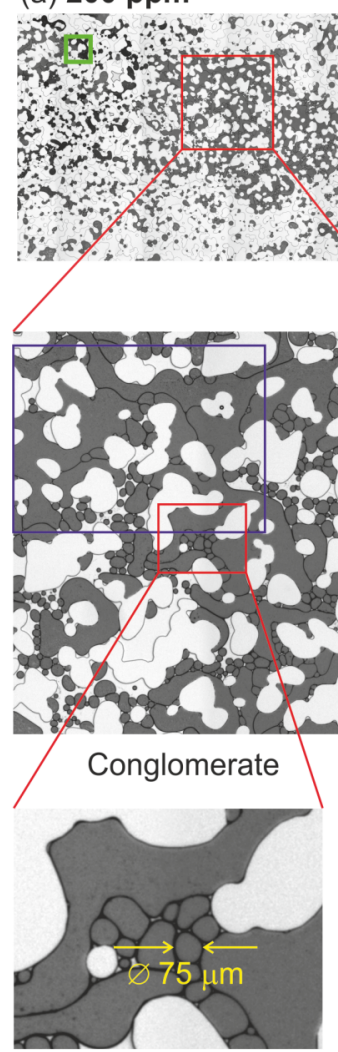

$\mathrm{O} / \mathrm{W}$ emulsion (b) $9000 \mathrm{ppm}$
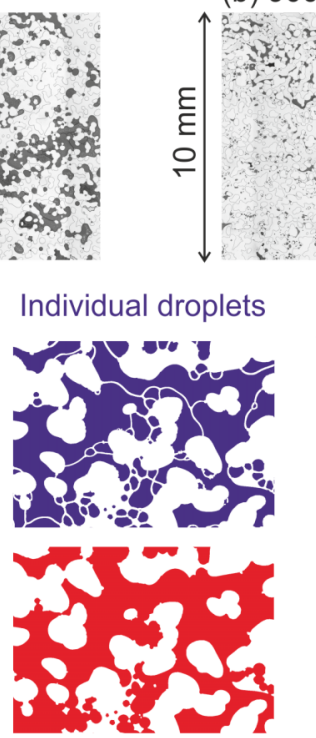

Connected phase
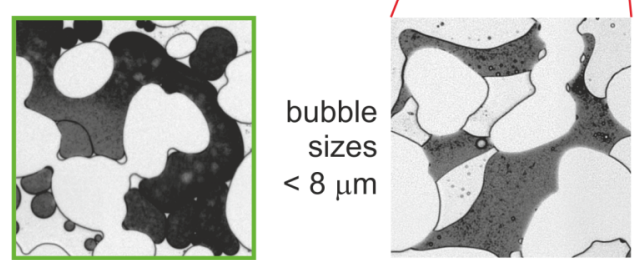

W/O emulsion optically not resolved

(c) $12000 \mathrm{ppm}$

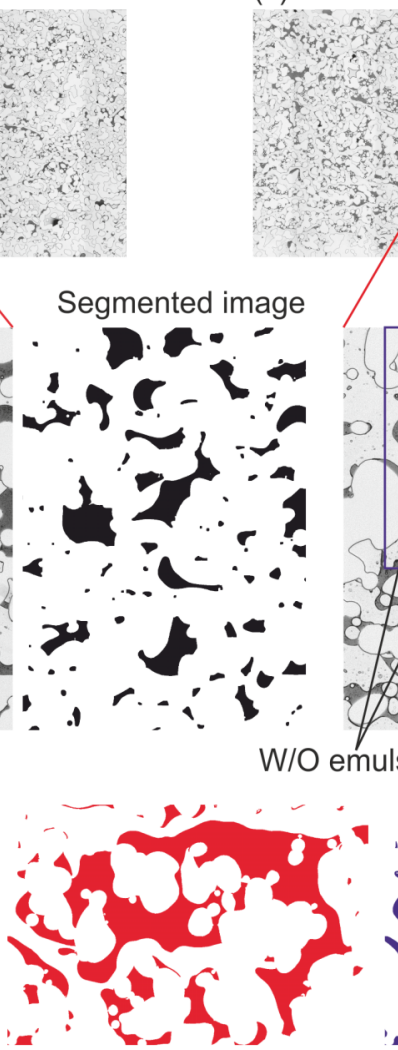

Connected phase

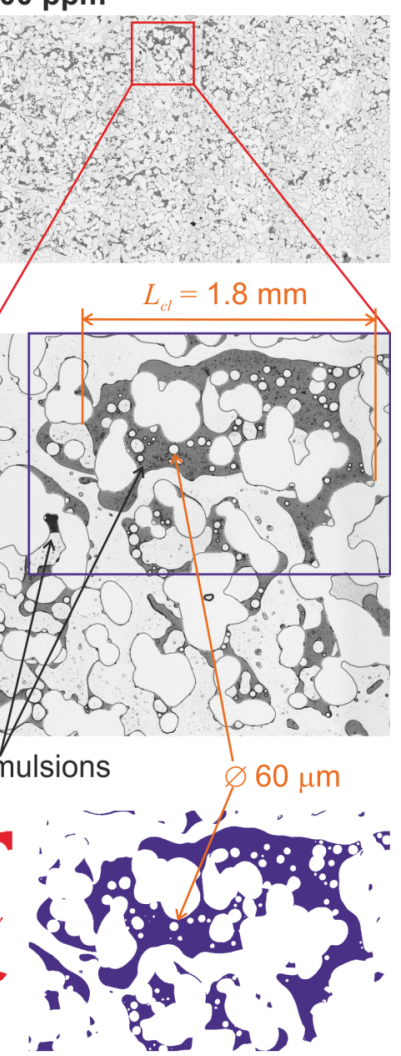

Individual droplets

FIG. 3. Aspects of the phase behavior under laminar flow conditions. Top row: total flooding domain after alkaline flooding with (a) 200, (b) 9000 , and (c) $12000 \mathrm{ppm} \mathrm{Na} \mathrm{CO}_{3}$. The zoom images below show details of oleic phase textures and examples of the respective segmented images: red: interpretation as connected emulsion phases (ignoring oleic-phase texture); blue: considering the oleic-phase texture. The different textures, respectively, phases, are discussed in the text.

are displayed as $C_{\mathrm{Na}_{2} \mathrm{CO}_{3}}=0$. The data show a characteristic behavior that will be linked to the displacement characteristics as discussed in the following.

The advantage of using MF is that it provides in principle all the information needed for a comprehensive evaluation of the saturation state and-as we will show-of flow processes. However, MF may not link directly to physical parameters, with which flow properties are commonly described, leading to a physical understanding of the displacement processes. In order to achieve a physical understanding, MF must be interpreted in terms of displacement physics. A first step has already been done by linking recovery - the produced oil volume- to the change of the area $A_{\text {oil }}$. In the next step, we calculate and interpret the Euler characteristic.

Euler characteristic. From Fig. 1, it becomes obvious that the wetting state of the sample changes during the experimental sequence. Initially, the system is in an oil-wet state. This is due to the fact that we start the experiments with $S_{o}=1$ and oil is initially wetting the entire glass surface. During water flooding, oil is partially displaced by water. In the swept areas, wettability changes to a more water-wet state; however, large clusters are still connected over several pores, partially covering the grains-we may call this a mixed-wet system or state. During alkaline flooding, larger clusters are pinned by their contacts to the grains, but successively break up by viscous forces into smaller nonwetting droplets; the system turns successively water wet where internal surfaces get in contact with the injection water.

It has recently been shown that the wetting state is closely linked to the Euler characteristic $(\chi)$, which describes the connectivity of a fluid phase [21]. In the following, we make use of this link to characterize the change of the wetting state during displacements. The Euler characteristic $\chi$ can be calculated from the Betti numbers, $\beta_{i}: \chi=\beta_{0}-\beta_{1}+\beta_{2}$. $\beta_{0}$ refers to the number of objects, i.e., the number of clusters in the system; $\beta_{1}$ is the number of loops or holes in the structure; and $\beta_{2}$ refers to the number of inclusions. In 2D systems, inclusions do not exist and $\beta_{2}=0$.

According to the definition, $\chi$ scales with the number of objects, and tiny droplets may contribute with the same weight as large clusters, which are more significant for oil production. Therefore $\chi$ is not a good measure in the present case, because recovery does not refer to the number of objects, but to oil volumes, and larger clusters contribute more than small droplets. We therefore propose an alternative Euler characteristic, $\chi_{v s}$, scaled by cluster volumes:

$$
\chi_{v s}=\frac{\sum_{n_{c l}} \chi_{c l} V_{c l}}{\sum_{n_{c l}} V_{c l}}
$$



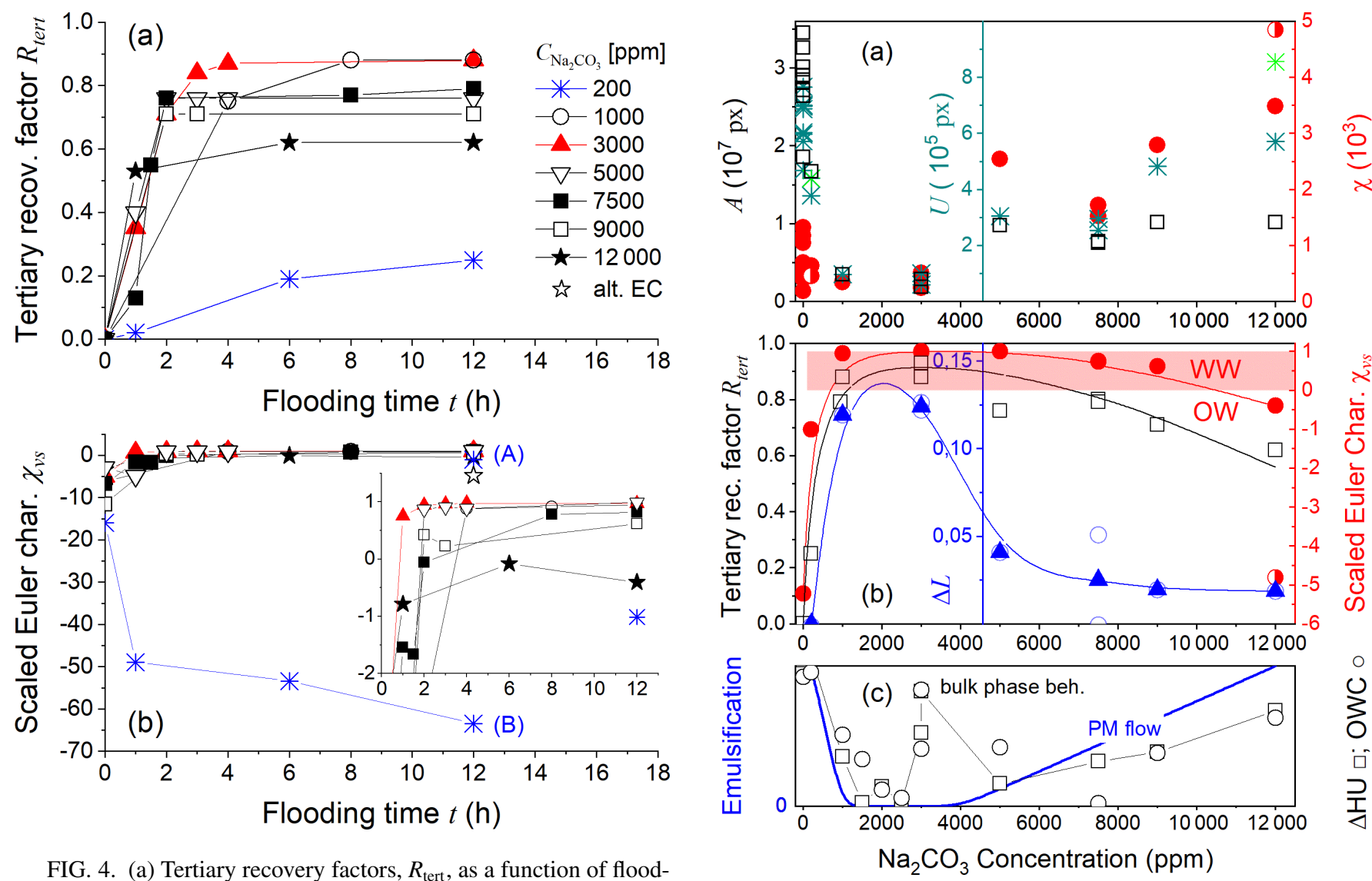

FIG. 4. (a) Tertiary recovery factors, $R_{\text {tert }}$, as a function of flooding time for different $\mathrm{Na}_{2} \mathrm{CO}_{3}$ concentrations. (b) The respective normalized Euler characteristic. (A) and (B) as well as $\xi$ and $\psi$ correspond to different interpretations of the 200-ppm and 12000 ppm experiment, respectively, as described in the text. The inset shows a zoon-in of the main panel.

with $n_{c l}$ being the number of clusters, $\chi_{c l}$ the Euler characteristic of an individual cluster, and $V_{c l}$ the volume of this cluster. As a result, the most water-wet state corresponds to individual droplets with $\chi_{c l}=1$ and has $\chi_{v s}=1$. The most oil-wet state then corresponds to $\chi_{v s}=1-N_{\text {grains }}$ with $N_{\text {grains }}$ being the number of "rock grains," which is then equal to the number of loops. The determination of $\chi_{c l}$ is illustrated in Fig. 1.

The evaluated $\chi_{v s}$ 's after the different water and alkaline floods have been plotted together with the recovery factors in Fig. 5(b). Referring to oil wet as $\chi_{v s}<0$ and to water wet as $\chi_{v s}>0$, we can state that after water flooding, generally an oil-wet behavior has been observed, changing toward a more water-wet state during alkaline flooding. This development$\chi_{v s}(t)$-is shown in the lower panel of Fig. 4. The exception for low alkali concentrations is a matter of phase behavior and will be discussed further below. For the range of favorable alkali concentrations (high recovery factor), the $\chi_{v s}$ indicates a strongly water-wet state.

For systems with a complex phase behavior, there may be more than one way to calculate the Euler characteristic. A good example is the 200-ppm case, for which formation of extensive conglomerates has been observed. Even appearing to the eye as individual droplets, conglomerates may behave as large clusters with a highly negative $\chi_{v s}$, even in cases that we would not call "oil wet." At that point, it is too simple to

FIG. 5. (a) 2D Minkowski functionals: area $A$, perimeter $U$, and Euler characteristic $\chi(a)$, and their interpretations (b) as a function of alkali concentration. and $*$ represent alternative descriptions of $\chi, \chi_{v s}$, and $U$ with respect to the oleic-phase texture. (b) The interpretations are the tertiary recovery factor $R_{\text {tert }}$, the volume-scaled Euler characteristics $\chi_{v s}$, and the difference in the Lorenz factor $\Delta L$ as calculated from the cluster volume distribution. (c) Degree of oil-phase swelling in bulk-phase experiments observed in CT scanning; $\Delta \mathrm{HU}$ denotes a normalized difference in gray-scale values of both phases, and OWC the level of the oil-water contact. All quantities are relative and derived from the data shown in Fig. 2. The qualitative degree of emulsification in porous media flow is plotted for comparison.

talk about a single connected cluster or individual droplets. It also may be too simple to link the Euler characteristic to the wetting state, but still reflect phase connectivity. In Fig. 4, the Euler characteristic for two alternative interpretations of the 200-ppm experiment is given; (A) accounts for individual droplets, even if they form conglomerates; (B) treats conglomerates as objects. Examples of both methods of image segmentation are given in Fig. 3. Conglomerates may be exceptional and leave room for interpretation with respect to cluster size distribution and $\chi_{v s}$, depending on whether they are accounted for as individual small clusters, or rather as a supercluster. In the present study, we interpret the Euler characteristic as individual droplets [Fig. 5(b)] referring to some extent to the wetting state, and for the cluster size distribution, we rather refer to phase connectivity and to the conglomerate as a unity. 

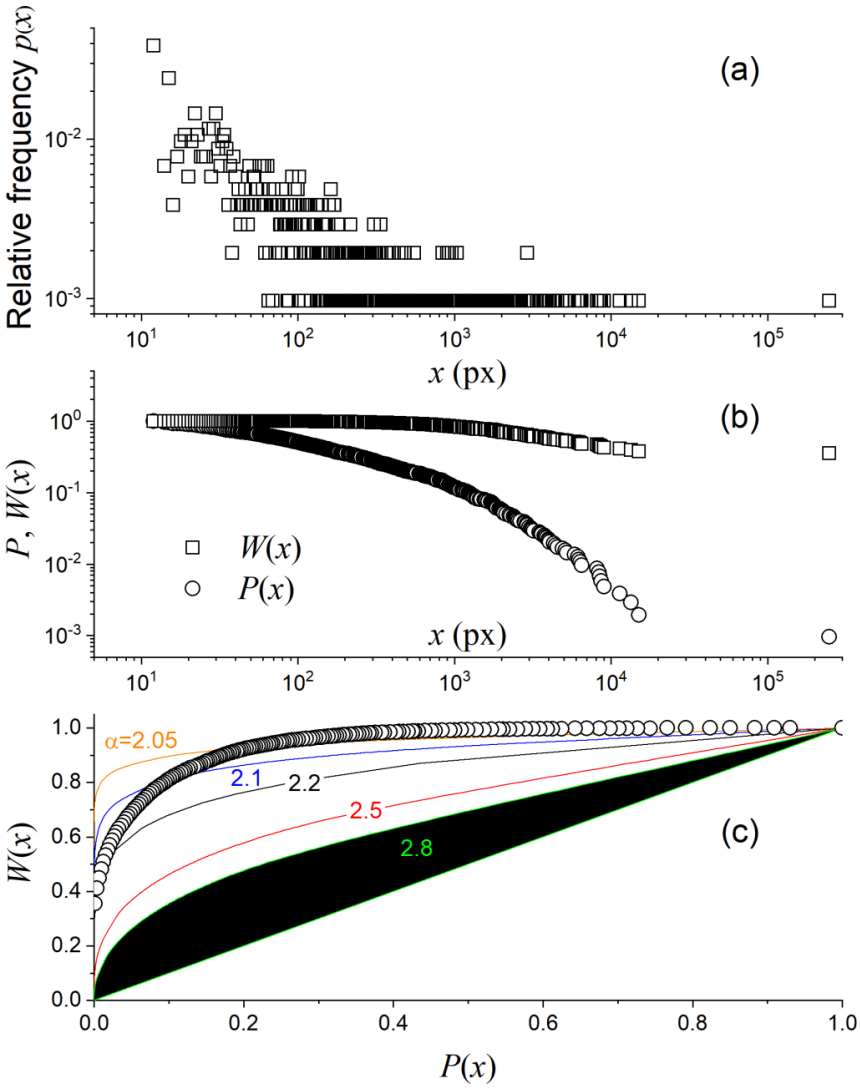

FIG. 6. Data were taken from a water flood. (a) Cluster volume distribution (CVD) $p(x)$ (relative frequency as a function of cluster size). (b) Cumulative CVD $P(x)$ and volume-weighted cumulative CVD $W(x)$. (c) Lorenz plot $W(P)$. For comparison, Lorenz plots for fractal distributions with different exponents $\alpha$ are given in the same panel. The area under the curves, as indicated for $\alpha=2.8$, is a measure of "heterogeneity." In the present case, $P(x)$ corresponds to the largest fraction of clusters, and $W(x)$ to the total oil volume associated with this fraction of clusters. For fractal distributions: $W=P^{(\alpha-2) /(\alpha-1)}$.

Lorenz analysis. The data presented so far indicate a plateau of the recovery factor and the Euler characteristic as a function of the alkali concentration. The optimum can be found in a range between 1000 and 3000 ppm $\mathrm{Na}_{2} \mathrm{CO}_{3}$ with a mild decline to higher concentrations. Both quantities are associated with uncertainties, which are a result of the investigated domain size versus the largest clusters, which are statistically not well represented in the domain.

In contrast to the recovery factor and the Euler characteristic, the displacement characteristic may be more robustly reflected in the cluster size distribution, which is less sensitive to individual clusters at the tail end of the distribution. Figure 6 illustrates various cluster-volume distribution plots as applied in the following to all data sets; it serves as a reference. The top row of Fig. 7 shows the cumulative cluster size distributions $P(x)$ at the end of the respective floods. The data were calculated from the cluster size distribution, $p(x)$, by integration $[33,34]$ :

$$
P(x)=\int_{x}^{\infty} p(y) d y .
$$

As a first observation, the results on water flooding (blue symbols) show a very consistent behavior despite the scatter in the recovery data and the Euler characteristic. Comparing the individual data sets to the subsequent alkaline floods, we find that $P(x)$ has a much narrower distribution to smaller clusters after alkaline flooding, consistent with the visual observations. Assuming for a moment a fractal distribution, $p(x)=c x^{-\alpha}$, an exponent $\alpha$ can be determined by a maximum likelihood approach [18,33,34]. In a double logarithmic plot, $\alpha$ appears as the slope of a straight line fit, which is shown as lines in the upper row of Fig. 7; we find that $\alpha$ becomes generally larger after alkaline flooding, consistent with a narrower cluster volume distribution (represented by the area in 2D), and deviates most strongly in cases where we find high recovery and positive $\chi_{v s}$ and is rather similar for the cases we consider outside the optimum. However, this signature is not very robust, because the range over which the data show fractal behavior is about one order of magnitude and hence rather limited.

It is evident that the size of an individual cluster directly refers to (a) phase mobility (cluster length) and to (b) recovery (cluster volume). Therefore, an imbalance of the cluster size distribution to larger clusters may be beneficial for the mobilization of oil; i.e., there is a higher EOR potential. Such an imbalance may be described by Lorenz diagrams. This concept refers back to Max Otto Lorenz (1880-1962), who was an American economist and described with his concept the distribution of wealth in society [35]; next to $P(x)$, we calculate the volume-weighted cluster size distribution by integration:

$$
W(x)=\frac{\int_{x}^{\infty} y p(y) d y}{\int_{x_{\min }}^{\infty} y p(y) d y},
$$

with $x_{\min }$ being the lower bound of the distribution. Plotting $W(P(x))$ results in the Lorenz plot. We translate the economic interpretation $-P$ being the richest fraction of the population and $W$ the associated fraction of the total wealth-to the problem of oil production $-P$ being the fraction of largest clusters and $W$ being the total volume of oil associated with this fraction of clusters. The closer the Lorenz curve tends to be a straight line (the diagonal), the more equally the oil is distributed over the different classes of clusters. The more the curve deviates from the diagonal to the upper left corner, the more the large clusters are dominating the oil volume-an imbalance of wealth.

We may associate larger clusters with higher EOR potential, since large clusters are more easily mobilized, or, in the present case, may be subject to cluster breakup by viscous forces. The lower row of Fig. 7 compares the Lorenz plots after water flooding and alkaline flooding. We observe in all cases (but 200 ppm, where no change has been observed) a deviation of the Lorenz curve to a more uniform distribution due to alkaline flooding. Together with the decrease of absolute cluster sizes, this indicates a decrease in further EOR potential. The signature is most pronounced in the range where highest recovery and highest Euler characteristic is observed. The effect of alkaline flooding in the Lorenz plots appears to be a robust indication of a working EOR process, which can be quantified by the difference of the Lorenz factor 

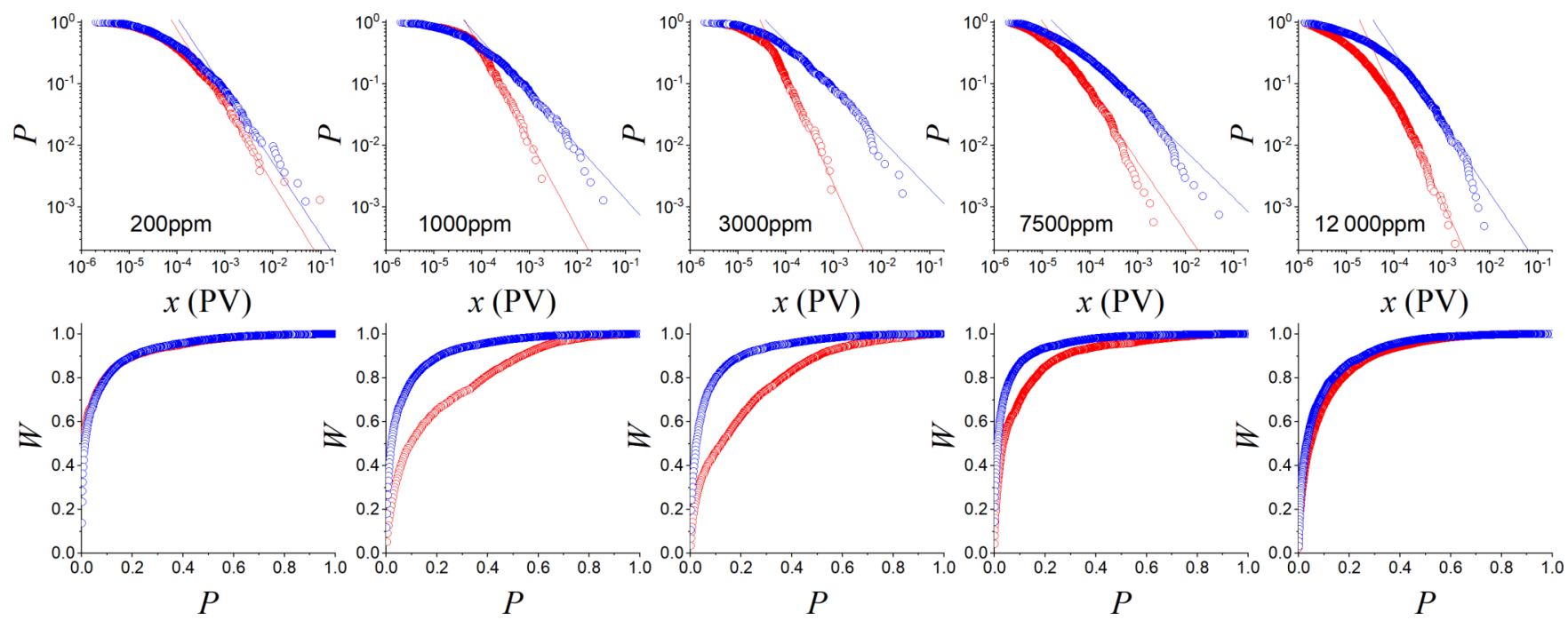

FIG. 7. Top row: cumulative cluster size distributions for three experimental sequences of water floods (blue) and alkaline floods (red) with 200, 1000, 3000, 7500, and $12000 \mathrm{ppm} \mathrm{Na}_{2} \mathrm{CO}_{3}$. The lines correspond to power law fits by a maximum likelihood approach. Bottom row: Lorenz plots for the same data sets.

of water flooding and alkaline flooding $\Delta L$, with

$$
\Delta L=2 \int_{0}^{1}\left(W_{\mathrm{WF}}-W_{\mathrm{AF}}\right) d P,
$$

which varies in between 0 at $200 \mathrm{ppm}$ and 0.12 at optimum performance. Its dependency on the $\mathrm{Na}_{2} \mathrm{CO}_{3}$ concentration is compared to the recovery factor and the Euler characteristic in Fig. 5; it shows a stronger decline to higher concentrations than the other quantities and therefore defines the optimum better.

\section{CONCLUSIONS AND DISCUSSIONS}

It is quite remarkable that in porous media flow emulsification was observed for high and low alkali concentrations, but not for concentrations in between that we consider as optimum condition; emulsification and the recovery factor behave nonmonotonically. This is essentially in line with bulkphase experiments; however, the degree of emulsion formation in porous media flow is minor compared to the bulk-phase behavior. Under laminar flow conditions emulsification may be limited by the limited contact area between the two phases during displacement, by the supply of these interfaces with the emulsifying agent, referring to the water-velocity field, and by the local mixing/transport mechanisms at the oil/water interface.

Emulsion phases are known to influence displacements due to various aspects; emulsions may facilitate ultralow interfacial tension between oil and brine, improving the displacement efficiency. On the other hand, emulsion phases typically have a substantially higher viscosity than their constituents, which may oppose displacements $[2,14]$. The direct link between the degree of emulsification and the displacement efficiency has been demonstrated in the present study.

A first attempt - a hypothesis - to understand the degree of emulsification requires consideration not only of the bulkphase behavior, but also of the flow regime: At low alkali concentrations, the improvement in displacement efficiency is small compared to the preceding water flood, and most oil clusters remain stagnant. The stagnant clusters are continuously contacted with the injected emulsifier, and over time, W/O emulsions form. Due to the increasing viscosity of the oleic phase, affected clusters remain stagnant even at lower IFT. Initially mobilized oil droplets/clusters adhere to each other and form conglomerates or lose $\mathrm{O} / \mathrm{W}$ emulsions remaining in the system; the resulting IFT is still too high to break up these conglomerates. At very high alkali concentrations, the wetting state tends to be more oil wet, which is probably due to the relatively high ionic strength (salinity) of the injection water. The oil phase adheres to the solids, without being globally connected. The high alkali concentration allows relatively fast emulsification; both effects limit oil phase mobility already at an early stage. For concentrations in between-under optimal conditions - already the bulk-phase behavior shows a minimum emulsion volume, predominantly in a water- and oil-external phase. Such a microemulsion may be limited to the oil-water interface (this has not been resolved in the present study) and may therefore be highly effective in mobilizing oil clusters. On the other hand, the salinity is still low enough to facilitate a favorable water-wet state.

However, despite the complexity of the phase behavior during displacements, the topological properties of the remaining oleic phase show trends as a function of alkali concentration, reflecting the nonmonotonic characteristic of the displacement and the phase behavior.

The difficulty of a topological description is its translation into a physical picture. With the interpretation of topology in terms of wettability, phase connectivity, recovery factor, and EOR potential, we extract additional information on the displacement process and quantify observations. What are the signatures of an ideal alkaline flood or-more general-an ideal EOR displacement? By changing the capillary number, larger clusters break up into smaller clusters. It has been observed that these smaller clusters are nonwetting and mobile. On this basis, we define the EOR potential: (1) the 
initial cluster size distribution (volume distribution) shows a strong imbalance toward large clusters. As a measure of this imbalance we use Lorenz diagrams and for quantification the Lorenz factor, and (2) in the present case, the large clusters are predominantly pinned to the solid phase, i.e., being in a wetting state. To analyze the wetting state, we introduced a volume-weighted Euler characteristic. These statistical and topological phase properties change from water flooding to the subsequent alkaline flooding; on this basis, we optimize the injection-water composition. In the present case, the Lorenz diagrams have shown the most significant signature. Other chemical methods can be expected to have their own "signature," e.g., low-salinity water flooding may mainly change the wetting state, but may not affect the cluster size distribution as much. We expect that in this case the Euler characteristic may be more indicative than the Lorenz diagrams.

\section{ACKNOWLEDGMENTS}

The authors acknowledge the OMV AG, Vienna, Austria and the FFG (Österreichische Forschungsförderungsgesellschaft) for supporting this project and especially Torsten Clemens for discussions on the problem statement. Furthermore, we would like to thank Ryan Armstrong for insightful discussions on fluid topology and Hassan Mahani, Steffen Berg, and Hendrik Rohler for commenting on the manuscript.
[1] C. N. Mulligan, R. N. Yong, and B. F. Gibbs, Surfactantenhanced remediation of contaminated soil: A review, Geoenviron. Eng. 60, 371 (2001).

[2] L. Lake, R. T. Johns, W. R. Rossen, and G. A. Pope, Fundamentals of Enhanced Oil Recovery (Society of Petroleum Engineers, Richardson, TX, 2014).

[3] S. Paria, Surfactant-enhanced remediation of organic contaminated soil and water, Adv. Colloid Interface Sci. 138, 24 (2008).

[4] C. C. West and J. H. Harwell, Surfactants and subsurface remediation, Environ Sci. Technol. 26, 2324 (1992).

[5] G. Hirasaki, C. A. Miller, and M. Puerto, Recent advances in surfactant EOR, SPE J. 16, 889 (2011).

[6] A. A. Olajire, Review of ASP EOR (alkaline surfactant polymer enhanced oil recovery) technology in the petroleum industry: Prospects and challenges, Energy 77, 963 (2014).

[7] T. S. Ramakrishnan and D. T. Wasan, A Model for interfacial activity of acidic crude oil/caustic systems for alkaline flooding, Soc. Pet. Eng. J. 23, 602 (1983).

[8] H. Mohammadi, M. Delshad, and G. A. Pope, Mechanistic modeling of alkaline/surfactant/polymer floods, SPE Reservoir Eval. Eng. 12, 518 (2009).

[9] T. R. French and T. E. Burchfield, Design and optimization of alkaline flooding formulations, SPE/DOE Enhanced Oil Recovery Symposium, 1990 (unpublished).

[10] S. Berg, R. T. Armstrong, A. Georgiadis, H. Ott, A. Schwing, R. Neiteler, N. Brussee, A. Makurat, M. Rucker, L. Leu, M. Wolf, F. Khan, F. Enzmann, and M. Kersten, Onset of oil mobilization and nonwetting-phase cluster-size distribution, Petrophysics 56, 15 (2015).

[11] M. Rücker et al., From connected pathway flow to ganglion dynamics, Geophys. Res. Lett. 42, 3888 (2015).

[12] S. Berg et al., Real-time 3D imaging of Haines jumps in porous media flow, Proc. Natl. Acad. Sci. USA 110, 3755 (2013).

[13] V. A. Lifton, Microfluidics: An enabling screening technology for enhanced oil recovery (EOR), Lab Chip 16, 1777 (2016).

[14] E. Unsal, M. Broens, and R. T. Armstrong, Pore scale dynamics of microemulsion formation, Langmuir 32, 7096 (2016).

[15] W.-B. Bartels et al., Oil configuration under high-salinity and low-salinity conditions at pore scale: A parametric investigation by use of a single-channel micromodel, SPE J. 22, 1362 (2017).
[16] M. Broens and E. Unsal, Emulsification kinetics during quasimiscible flow in dead-end pores, Adv. Water Resour. 113, 13 (2018).

[17] S. Iglauer, S. Favretto, G. Spinelli, G. Schena, and M. J. Blunt, $\mathrm{X}$-ray tomography measurements of power-law cluster size distributions for the nonwetting phase in sandstones, Phys. Rev. E 82, 056315 (2010).

[18] A. Georgiadis, S. Berg, A. Makurat, G. Maitland, and H. Ott, Pore-scale micro-computed-tomography imaging: Nonwettingphase cluster-size distribution during drainage and imbibition, Phys. Rev. E 88, 033002 (2013).

[19] R. T. Armstrong, A. Georgiadis, H. Ott, D. Klemin, and S. Berg, Critical capillary number: Desaturation studied with fast X-ray computed microtomography, Geophys. Res. Lett. 41, 55 (2014).

[20] R. T. Armstrong, J. E McClure, M. A. Berrill, M. Rucker, S. Schlüter, and S. Berg, Beyond Darcy's law: The role of phase topology and ganglion dynamics for two-fluid flow, Phys. Rev. E 94, 043113 (2016).

[21] R. T. Armstrong, J. E. McClure, V. Robins, Z. Liu, C. H Arns, S. Schlüter, and S. Berg, Porous media characterization using Minkowski functionals: Theories, applications and future directions, Transp. Porous Media 130, 305 (2019).

[22] J. E. McClure, R. T. Armstrong, M. A. Berrill, S. Schlüer, S. Berg, W. G. Gray, and C. T. Miller, Geometric state function for two-fluid flow in porous media, Phys. Rev. Fluids 3, 084306 (2018).

[23] Z. Liu, A. Herring, C. Arns, S. Berg, and R. T. Armstrong, Pore-scale characterization of two-phase flow using integral geometry, Transp. Porous Media 118, 99 (2017).

[24] K. R. Mecke, Additivity, Convexity, and Beyond: Applications of Minkowski Functionals in Statistical Physics (Springer, Berlin, Heidelberg, 2000), pp. 111-184.

[25] H. H. Khanamiri, C. F. Berg, P. A. Slotte, S. Schlüter, and O. Torsæter, Description of free energy for immiscible two-fluid flow in porous media by integral geometry and thermodynamics, Water Resour. Res. 54, 9045 (2018).

[26] H. Ott, A. Kharrat, M. Borji, T. Clemens, and P. Arnold Screening of EOR potential on the pore scale by statistical and topological means, International Symposium of the Society of Core Analysts, 2019 (unpublished).

[27] T. Amirian, M. Haghighi, and P. Mostaghimi, Pore scale visualization of low salinity water flooding as an 
enhanced oil recovery method, Energy Fuels 31, 13133 (2017).

[28] T. Amirian and M. Haghighi, Impact of clay type and water composition on low salinity water injection-visualisation approach, APPEA J. 58, 51 (2018).

[29] D. G. Avraam and A. C. Payatakes, Flow regimes and relative permeabilities during steady-state two-phase flow in porous media, J. Fluid Mech. 293, 207 (1995).

[30] S. L. Kokal, Crude oil emulsions: A state-of-the-art review, SPE Prod. Facil. 20, 5 (2005).

[31] Youssef, E. Rosenberg, H. Deschamps, R. Oughanem, E. Maire, and R. Mokso, Oil ganglia dynamics in natural porous media during surfactant flooding captured by ultra-fast $\mathrm{x}$-ray microtomography, International Symposium of the Society of Core Analysts, 2014 (unpublished).

[32] K. Michielsen and H. De Raedt, Integral-geometry morphological image analysis, Phys. Rep. 347, 461 (2001).

[33] A. Clauset, C. R. Shalizi, and M. E. J. Newman, PowerLaw Distributions in Empirical Data, SIAM Rev. 51, 661 (2009).

[34] Y. Virkar and A. Clauset, Power-law distributions in binned empirical data, Ann. Appl. Stat. 8, 89 (2014).

[35] M. O. Lorenz, Methods of measuring the concentration of wealth, J. Am. Stat. Assoc. 9, 209 (1905). 